\section{A pilot study of deep sclerectomy with implantation of chromic suture material as a collagen implant: medium-term results}

\begin{abstract}
Aims A pilot study of the safety and efficacy of deep sclerectomy with a chromic suture implant (DSCI) for open angle glaucoma. Methods This prospective trial involved 23 eyes of 16 consecutive patients with uncontrolled open angle glaucoma. The DSCI procedure involved radial suturing of a $2 \mathrm{~mm}$ piece of 1/0 chromic suture as an implant. Intraocular pressure (IOP) measurements and eye examinations were performed preoperatively and regularly postoperatively for up to 66 months.
\end{abstract}

Results The mean follow-up period was 36.3 (SD 19.9) months. The mean preoperative IOP was 26.3 (SD 8.5) $\mathrm{mmHg}$. The mean postoperative IOP was 14.1 (SD 2.8) $\mathrm{mmHg}$ at month 12, and 16.5 (SD 2.3) $\mathrm{mmHg}$ at 36 months. Complete success rate, defined as an IOP lower than $21 \mathrm{mmHg}$ without medication was $77 \%$ of eyes at 36 months. Qualified success rate, defined as an IOP lower than $21 \mathrm{mmHg}$ with medication, was $100 \%$ at 36 months. Paired $t$-test and Wilcoxon test analysis of preoperative $v s$ postoperative IOP at 36 months indicated a significant reduction in IOP. Intraoperative complications included rupture of the trabecular meshwork in seven eyes. Exclusion of these patients from the results analysis revealed a mean follow-up of 37.1 months (SD 9), preoperative mean IOP was $25.9 \mathrm{mmHg}$ (SD 4.1), 12-month mean IOP was $13.4 \mathrm{mmHg}$ (SD 2.8), and 36-month mean IOP $16.6 \mathrm{mmHg}$ (SD 2.1). Early postoperative complications included hyphaema in two eyes and dislocation of implant in one eye. One eye developed a central retinal vein occlusion and cataract progression occurred in one eye.
MT Wevill', D Meyer ${ }^{1}$ and E Van Aswegen ${ }^{1}$

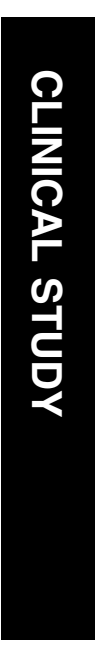

The mean postoperative duration of use of topical dexamethasone was 5.4 months (SD 2). Conclusions DSCI using a chromic suture implant provides good control of IOP at medium-term follow-up with few postoperative complications. Prolonged use of postoperative anti-inflammatory medication was necessary.

Eye (2005) 19, 549-554. doi:10.1038/sj.eye.6701541

Published online 10 December 2004

Keywords: deep sclerectomy; filtering surgery; open angle glaucoma

\section{Introduction}

A number of nonpenetrating glaucoma procedures have been described for the surgical treatment of medically uncontrolled open angle glaucoma. ${ }^{1-15}$ In 1989, Fyodorov, Kozlov and colleagues described a nonpenetrating deep sclerectomy procedure with implantation of a collagen drainage device. The Aqua-Flow ${ }^{\mathrm{TM}}$ collagen drainage device (STAAR Surgical Company) is placed under a scleral flap and facilitates the flow of aqueous humour out of the eye to form a bleb under the conjunctiva. Demailly, Karlen and others have published medium- to long-term results of this procedure, which is successful in lowering intraocular pressure (IOP) and is reported to have a lower rate of postoperative complications than standard trabeculectomy. ${ }^{7-12}$ There is a lower risk of postoperative inflammation and shallow anterior chambers. However, the surgical technique is more difficult than standard trabeculectomy and the implant is expensive. Other implants including the T-Flux ${ }^{13}$
${ }^{1}$ Department of

Ophthalmology,

Tygerberg Hospital and University of Stellenbosch, Cape Town, South Africa Aston Academy of Life Sciences, Aston University, Birmingham B4 7ET

E-mail:mail@

wevs.fslife.co.uk

Received: 14 June 2003 Accepted: 10 March 2004 Published online: 10 December 2004

The authors have no proprietary interests in any of the products mentioned.
Correspondence: MT Wevill, 
(a nonabsorbable implant) and SKGEL ${ }^{\circledR 14,15}$

(a reticulated hyaluronic acid implant) have also been developed for nonpenetrating filtering surgery.

The Aqua-Flow ${ }^{\mathrm{TM}}$ collagen drainage device consists of lyophilized porcine scleral collagen, which is altered to delay absorption. The dry dimensions of the implant are $1 \times 1 \times 2.5 \mathrm{~mm}$. The implant swells with aqueous and tissue fluid once it has been placed in the eye and it is absorbed within approximately 6-9 months. ${ }^{16}$ A $3 \mathrm{~mm}$ piece of $\mathrm{O}$ chromic suture (Ethicon) was implanted in eyes in our study to evaluate the procedure in a population of white and mixed race patients with medically uncontrolled open angle glaucoma. This implant has dry dimensions of $0.5 \mathrm{~mm} \times 0.5 \mathrm{~mm} \times 3 \mathrm{~mm}$ and also swells once hydrated. It consists of collagen from sheep or cow intestinal submucosa and serosa. It is altered by chromicizing to delay absorption and it has been safely used in the human body for many years. The advantages of this implant are its low cost and that it may be used in patients with religious objections to the use of porcine products. In this study, we analysed the outcome and complications of the first 23 eyes that underwent deep sclerectomy with chromic suture implant (DSCI) and were followed for up to 66 months.

\section{Materials and methods}

The Ethics Committee of the University of Stellenbosch, South Africa, approved the prospective study, and informed consent was obtained from all the patients. Inclusion criteria were primary or secondary open angle glaucoma uncontrolled by medical therapy, age over 40 years, no previous ocular surgery and no previous intraocular inflammation. Uncontrolled glaucoma was defined as an uncontrolled IOP with maximal tolerable antiglaucoma medical therapy with evidence of progression of the optic neuropathy and/or a visual field defect. Exclusion criteria were angle closure glaucoma, refusal to participate in the study, and previous eye surgery or previous argon laser trabeculoplasty.

Before surgery, all patients had a full eye history and examination. The IOP was measured with a Goldmann applanation tonometer, and 24-2 threshold visual fields were done on each patient with a Humphrey visual field analyser. After surgery, eye examinations were conducted on days 1 and 7, and at months 1, 2, 3, 6, 9, 12, and 6 monthly thereafter. More frequent examinations were carried out if indicated. All postoperative assessments were by the surgeon (MW) or a registrar. All complications were recorded. The anterior chamber was recorded as shallow if there was peripheral iridocorneal touch and flat if there was lens corneal touch. Cataract was defined as deterioration in visual acuity attributable to an increase in lens opacity.
A similar surgical technique was performed on all the patients by the same surgeon (MW). Peribulbar anaesthesia using 3-5 ml of a mixture of $30 \%$ xylocaine $2 \%$ and $70 \%$ bupivacaine $0.75 \%$ and hyaluronidase $50 \mathrm{U}$ was given to each patient. A superior rectus muscle suture was placed. A fornix-based conjunctival flap was raised. A $4 \times 4 \mathrm{~mm}$ scleral flap, approximately $200 \mu \mathrm{m}$ thick was dissected into clear cornea. A $200 \mu \mathrm{m}$ deep sclerectomy exposed the canal of Schlemm and which was deroofed and the dissection continued anteriorly and down to Descemet's membrane. A 3-mm piece of 0 chromic was sutured into the scleral bed with $10 / 0$ nylon. Both the sclera and the conjunctiva were closed with 10/0 nylon. No intraoperative antimetabolites were used. Postoperatively patients were treated with topical dexamethasone $0.1 \%$ and chloramphenicol $0.5 \%$ (Spersadex Comp $^{\mathrm{TM}}$, Restan) three times a day for 1 month, then switched to dexamethasone $0.1 \%$ (Spersadex $^{\mathrm{TM}}$, Restan) until the bleb inflammation settled. If the bleb appeared to be very inflamed despite topical dexamethasone, $1 \mathrm{ml}$ betamethasone $0.3 \%$ (Celestone Soluspan $^{\mathrm{TM}}$, Schering-Plough) was injected subconjunctivally. If the subconjunctival injection of betamethasone failed to control the inflammation, subconjunctival mitomycin C $0.02 \% 0.1 \mathrm{ml}$ was injected.

When the trabecular meshwork was penetrated during surgery, the procedure was converted to a standard trabeculectomy but the patient was not excluded from the study. Surgery was considered a complete success when IOP was less than or equal to $21 \mathrm{mmHg}$ without glaucoma medication, a qualified success when IOP was less than or equal to $21 \mathrm{mmHg}$ with glaucoma medication, and a failure when IOP was greater than $21 \mathrm{mmHg}$ with or without glaucoma medication. An eye was withdrawn from the study if any further surgery (including glaucoma drainage surgery) was performed on the eye, and follow-up of an eye ceased if a patient died or failed to attend follow-up appointments. All complications were noted.

Goniopuncture with the Nd:YAG laser was performed through a gonio lens when the filtration through the trabeculo-Descemet's membrane was insufficient. Results were analysed using the Student's $t$-test and Wilcoxon tests.

\section{Results}

In total, 23 eyes of 16 patients with uncontrolled primary and secondary open angle glaucoma had DSCI over a period of 9 months by a single surgeon (MW). All patients were enrolled consecutively. There were three male and 13 female patients; and five white patients and 11 patients of mixed race. The mean age was 66.6 years (SD 10.1 years) (See Table 1). 
Table 1 Patient data (23 eyes, 16 patients)

\begin{tabular}{lr}
\hline Mean age (years) & 66.6 \\
Male/female & $3 / 13$ \\
Mixed race/White & $11 / 5$ \\
Preoperative IOP (mmHg) & 26.3 \\
Follow-up (months) & 37.4 \\
\hline
\end{tabular}

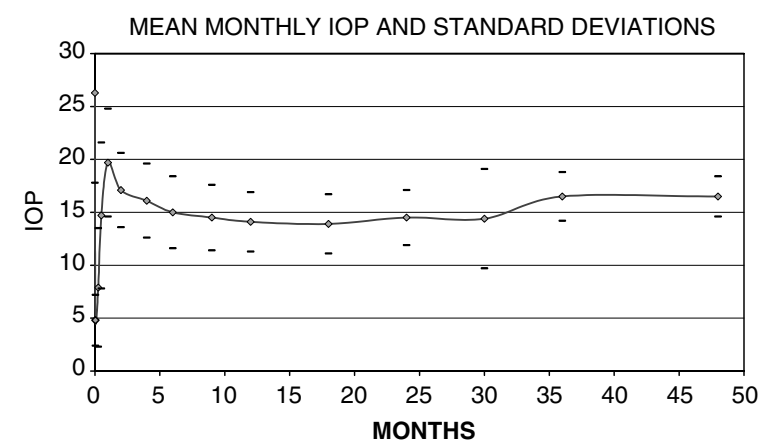

Figure 1 IOP before and after deep sclerectomy with chromic implant.

The mean follow-up time was 36.3 months (SD \pm 19.9 months). The mean preoperative IOP was $26.3 \mathrm{mmHg}(\mathrm{SD} \pm 8.5 \mathrm{mmHg})$. The mean postoperative IOP on the first postoperative day was $4.8 \mathrm{mmHg}$ $(\mathrm{SD} \pm 2.4 \mathrm{mmHg}), 14.1 \mathrm{mmHg}(\mathrm{SD} \pm 2.8 \mathrm{mmHg})$ after 12 months, and $16.5 \mathrm{mmHg}(\mathrm{SD} \pm 2.3 \mathrm{mmHg})$ after 36 months. The average IOP at 12 months was reduced by $46.4 \%$, and by $37.3 \%$ at 36 months (Figure 1). Eyes that underwent DSCI and remained in the study had a complete success rate of $77 \%$ at 36 months; the qualified success rate was $100 \%$ at 36 months.

Seven patients $(30 \%)$ had perforation of their trabecular meshworks intraoperatively. When these patients are excluded, the results are as follows. The mean follow-up period is 37.1 months (SD 9.0), the mean preoperative IOP was $25.9 \mathrm{mmHg}$ (SD $4.1 \mathrm{mmHg}$ ), the mean day 1 postoperative IOP was $5.1 \mathrm{mmHg}$ (SD $2.5 \mathrm{mmHg}$ ), the mean month 12 IOP was $13.4 \mathrm{mmHg}$ (SD $2.8 \mathrm{mmHg}$ ), and the mean month 36 IOP was $16.8 \mathrm{mmHg}$ (SD $2.1 \mathrm{mmHg}$ ). The complete success rate was $80 \%$ at 36 months and qualified success rate was $100 \%$.

The paired $t$-test and the Wilcoxon test of preoperative vs postoperative IOP after 36 months indicate that there is a significant reduction in IOP in the analysis of all patients and analysis of the DSCI patients only. The paired $t$-test $P$-value is 0.001 and for the Wilcoxon test the $P$-value is 0.005 for both groups.

Postoperative complications that occurred are listed (Figure 2). There were no cases of flat anterior chamber, no bleb- or surgery-related endophthalmitis. Two eyes, both of which had DSCI developed small postoperative

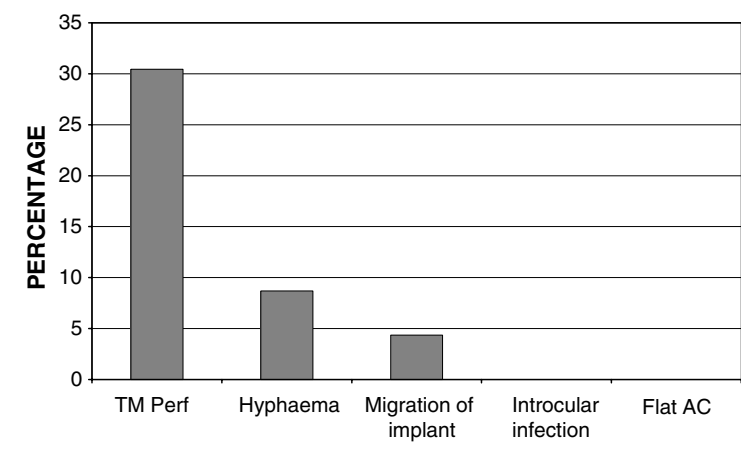

Figure 2 Postoperative complications.

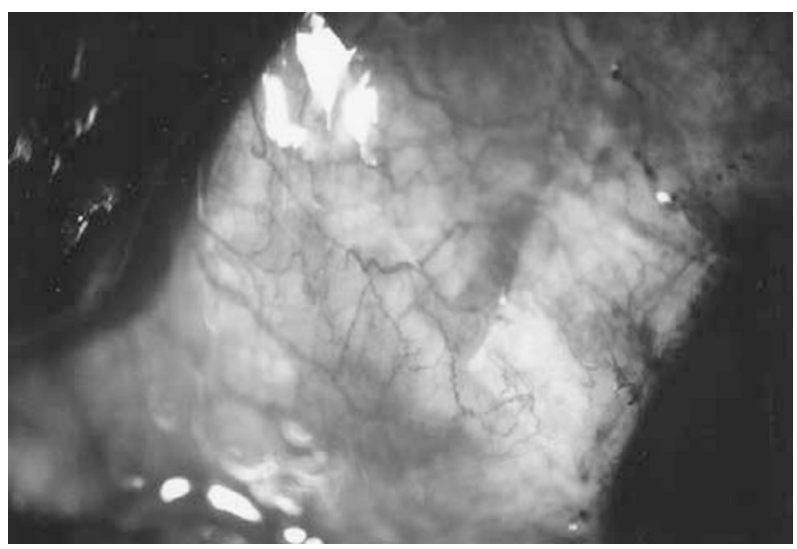

Figure 3 Chronic suture under the scleral flop (day 2).

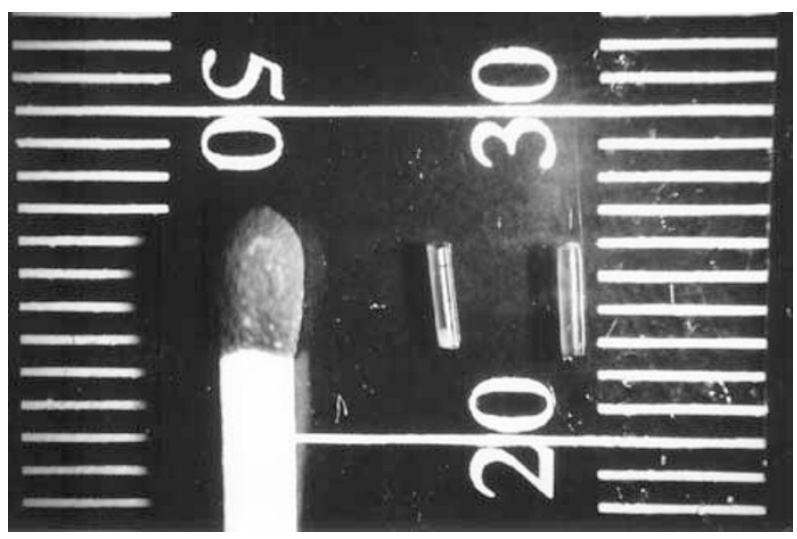

Figure 4 Dehydrated 0 chronic suture (on left) and hydrated 0 chronic suture (on right).

hyphaemas ( $12.5 \%$ of DSCI eyes), one eye ( $4 \%$ ) developed a visually significant cataract 7 months after the procedure (a trabeculectomy eye) (Figure 3) and one eye developed a central retinal vein occlusion (DSCI eye) (Figure 4). In one eye, the implant was dislodged through the trabecular meshwork into the anterior chamber after blunt trauma to the eye 3 weeks after the surgery (DSCI 
eye). This patient had corneal oedema and anterior segment inflammation. The implant was removed, and the oedema and inflammation settled within 3 weeks.

Bleb inflammation was treated with topical dexamethasone $0.1 \%$ (Spersadex ${ }^{\mathrm{TM}}$, Restan). The mean duration of dexamethasone use was 5.4 months (SD 2 months). Betamethasone $0.3 \%$ (Celestone Soluspan ${ }^{\mathrm{TM}}$, Schering-Plough) was injected subconjunctivally in 10 eyes (43\%). The mean time between operation and first subconjunctival injection was 4 weeks.

Subconjunctival mitomycin C $0.02 \% 0.1 \mathrm{ml}$ was injected in six patients (26\%) at an average of 2 months postoperatively and only one injection was administered in any eye treated.

Goniopuncture with the Nd:YAG laser was performed on five eyes (22\%). The mean time between operation and goniopuncture was 4 weeks.

\section{Discussion}

Daily medical glaucoma therapy is expensive ${ }^{17}$ and subject to poor patient compliance. ${ }^{18}$ Early surgery (by trabeculectomy) results in lower IOPs and better visual field preservation than medical and laser therapy. ${ }^{19}$ South Africa has limited health care funding. Therefore, the ideal glaucoma treatment should be effective, safe, and inexpensive. Different nonpenetrating filtering surgery techniques have been devised. Zimmerman, Arenas, Chiselta, and Stegmann have reported good results with a non penetrating procedure without an implant. ${ }^{2,4,5,12}$ Chiselta found a lower success rate than trabeculectomy using no implant. To improve results, various devices have been developed for implantation under a scleral flap including collagen implants such as the Aqua-Flow ${ }^{\mathrm{TM}}$ collagen drainage device, $\mathrm{T}-\mathrm{Flux}^{13}$ (a nonabsorbable implant) and SKGEL ${ }^{\mathbb{1} 14,15}$ (a reticulated hyaluronic acid implant). The Aqua-Flow ${ }^{\mathrm{TM}}$ is made of lyophilized porcine collagen that has been treated to delay absorption. Kozlov, Fyodorov, Mermoud, Demailly, and others have published excellent results using this implant. ${ }^{6-10}$ The success rate of DSCI with the AquaFlow $^{\mathrm{TM}}$ is comparable to trabeculectomy. ${ }^{20,21}$ Mermoud and co-workers and El Sayyad and co-workers found a similar success rate to trabeculectomy using the AquaFlow $^{\mathrm{TM}}$ implant. There is little comparative information available on the different implants and procedures.

The success rates of different nonpenetrating filtering surgery techniques vary because of differences in the study populations, different surgical techniques, postoperative fibrosis modulation, implants and surgeon experience. Nonpenetrating procedures take longer to perform and require greater skill than a trabeculectomy and the implants are an additional expense. However, there are advantages to nonpenetrating procedures.
Nonpenetrating procedures have a similar or lower incidence of postoperative complications s $^{12,20,21}$ compared to trabeculectomy. Therefore this pilot study was designed to determine whether a nonpenetrating procedure utilising chromic catgut was safe and effective for treating open angle glaucoma in patients attending the glaucoma clinic of Tygerberg Academic Hospital. A $3 \mathrm{~mm}$ piece of $1 / 0$ chromic catgut is significantly cheaper and was more readily available than the commercially produced collagen implant.

The high rate of perforation of the trabecular meshwork (seven out of 23 eyes, 30\%) in this study is as a result of the technical difficulty of the procedure, a lack of specific instruments for the procedure (steel blades with a standard trabeculectomy tray of surgical instruments was used and there were no nonpenetrating surgery-specific instruments available) and the inexperience of the surgeon. The 23 eyes enrolled in this study were the first 23 nonpenetrating procedures performed by the surgeon. Sanchez et $a l^{22}$ noted that scleral perforations occurred mainly during the learning phase of this surgical technique. In other studies the rate of trabecular meshwork perforation of surgeons learning the technique varied from $8.3 \%$ to $17 \%$ and decreases with surgeon experience. ${ }^{7}$ The success rate of DSCI with perforation and conversion to trabeculectomy is similar to planned trabeculectomy. ${ }^{21}$ The purpose of the study was to assess the outcome of deep sclerectomy with the chromic suture implant in the setting of a South African hospital. Therefore, consecutive cases of patients on whom this surgery was performed were reported and cases converted to trabeculectomy were included in the study. This would more accurately reflect the outcome of this surgery in the setting of an academic, training hospital, where there are a number of surgeons in training and therefore a high number of trabecular meshwork perforations can be expected. However, perforation of the trabecular meshwork could have affected the outcomes analysis in this study; therefore, the results were analysed including and excluding the trabecular meshwork perforation eyes. YAG laser goniopuncture was performed on some eyes. In these eyes the implant was left in situ and the laser meshwork perforations are microscopic compared to a classical trabeculectomy scleral excision. Therefore, these eyes are considered to have had nonpenetrating procedures for the purpose of this study.

There is variation in absorption rates of different brands of chromic catgut. ${ }^{23}$ In this study, Ethicon 1/0 chromic catgut was used. Lawrie ${ }^{24}$ studied the absorption of $3 / 0$ and $5 / 0$ catgut and chromic catgut implanted into the lumbar muscles of Wistar albino rats and demonstrated that the catgut is resorbed by a pyogenic reaction. Chromic catgut produces less of a 
pyogenic reaction than plain catgut and absorption takes longer with chromic catgut. The reaction consists of an early inflammatory reaction of polymorphonuclear leucocytes, which is maximal at 3 days. This reaction can be attributed to the surgical trauma of implanting catgut. By the 10th day a giant cell foreign body reaction develops. The chromic catgut is completely absorbed by the 16th day and the foreign body giant cell reaction continues until the 99th day, ie, the foreign body giant cell reaction persists after the absorption of the chromic catgut. $^{24}$ The same reaction may take place when using lyophilized porcine scleral collagen or other implants.

In this study, thicker chromic was used than in Lawrie's study. In some eyes, the chromic catgut was visible under the conjunctiva and Tenon's capsule for up to 12 months after the procedure. Slower absorption of 0 chromic catgut may occur because this suture is thicker than the 3/0 and 5/0 suture studied in Lawrie's study, and the scleral tissue is less vascular than skeletal muscle. In addition, after the nonpenetrating procedure topical steroids were directly applied to the conjunctiva to suppress subconjunctival inflammation and fibrosis. However, if a foreign body reaction persists after the absorption of the chromic implant, this may affect the success rate of the nonpenetrating procedure. The nature and duration of the inflammatory reaction in patients who have had a nonpenetrating procedure with an implant needs to be evaluated. If there is a long-term foreign body giant cell reaction in patients who have a nonpenetrating procedure, these patients may need to be on long-term immunosuppressants or antifibrotic agents to improve the success rate of the procedure. The long duration of dexamethasone treatment required by the patients in this study (an average of 5.4 months) indicates that there was a prolonged, visible inflammatory reaction in eyes receiving this collagen implant.

The prolonged inflammatory response and the associated increased treatment costs were interesting findings of the study especially in the context of trying to find a less expensive but effective surgical procedure for our glaucoma patients. The chromic suture was known to be cheaper than the commercially available implants, but the prolonged use of topical steroids and increased number of postoperative consultations reduces this benefit. In addition, the frequent follow-up appointments and prolonged use of eye medication required by these patients had implications for their quality of life.

This pilot study suggests that the DSCI procedure using chromic catgut as an intrascleral implant is safe and effective for the surgical treatment of low-risk open angle glaucoma patients. However, this is an uncontrolled study with a relatively small number of patients, and a medium-term follow-up period. Further controlled prospective studies are needed.

\section{Acknowledgements}

We acknowledge the support of the University of Stellenbosch for this study and thank Professor D Nel for the statistical analysis.

\section{References}

1 Krasnov MM. Sinusotomy: foundations, results, prospects. Trans Am Ophthalmol Otolaryngol 1972; 76: 369-374.

2 Zimmerman TJ, Kooner KS, Ford VJ, Olander KW, Mandlekorn RM, Rawlings EF et al. Trabeculectomy vs non-penetrating trabeculectomy: a retrospective study of two procedures in phakic patients with glaucoma. Ophthalmic Surg 1984; 15: 734-740.

3 Fyodorov SN. Non penetrating deep sclerectomy in open angle glaucoma. Eye Microsurg 1989; 3: 52-55 (in Russian).

4 Arenas E. Trabeculectomy ab-externo. Highlights Ophthalmol 1991; 19: 59-66.

5 Stegmann RC. Visco-canalostomy: a new surgical technique for open angle glaucoma. An Inst Barraquer (Spain) 1995; 25: 229-232.

6 Kozlov VI, Bagrov SN, Anisimova SY, Osipov AV, Mogilevtsev VV. Nonpenetrating deep sclerectomy with collagen. Eye Microsurg 1990; 3: 44-46 (in Russian).

7 Karlen ME, Sanchez E, Schnyder CC, Sickenberg M, Mermoud A. Deep sclerectomy with collagen implant: medium term results. Br J Ophthalmol 1999; 83: 6-11.

8 Demailly P, Jeanteur-Lunel MN, Berkani M, Ecoffet M, Kopel J, Kretz G et al. Non penetrating deep sclerectomy associated with collagen device in primary open angle glaucoma. Middle-term retrospective study. J Fr Ophthalmol 1996; 19(11): 659-666.

9 Shaarawy T, Karlen M, Schnyder C, Achache F, Sanchez E, Mermoud A. 5 year results of deep sclerectomy with collagen implant. J Cataract Refract Surg 2001; 27(11): 1770-1778.

10 Kazakova D, Roters S, Schnyder CC, Achache F, JonescuCuypers C, Mermoud A et al. Ultrasound biomicroscopy images: long-term results after deep sclerectomy with collagen implant. Graefes Arch Clin Exp Ophthalmol 2002; 240(11): 918-923.

11 Wishart PK, Wishart MS, Porooshani H. Viscocanalostomy and deep sclerectomy for the surgical treatment of glaucoma: a long term follow-up. Acta Ophthalmol Scand 2003; 81(4): 343-348.

12 Chiselta D. Non-penetrating deep sclerectomy versus trabeculectomy in primary open-angle glaucoma surgery. Eye 2001; 15: 197-201.

13 Ates H, Uretmen O, Azarsiz SS. Deep sclerectomy with a nonabsorbable implant (T-Flux): preliminary results. Can J Ophthalmol 2003; 38(6): 482-488.

14 Detry-Morel M, De Temmerman S. Assessment of nonpenetrating deep sclerectomy with reticulated hyaluronic acid implant SKGEL and/or preoperative application of 5-fluorouracil: results of 2 and a half years. Bull Soc Belge Ophthalmol 2003; 287: 53-62.

15 Luke C, Dietlein TS, Jacobi PC, Konen W, Krieglstein GK. A prospective randomised trial of viscocanalostomy with and without implantation of a reticulated hyaluronic acid implant (SKGEL) in open angle glaucoma. Br J Ophthalmol 2003; 87(5): 599-603. 
16 Chiou AGY, Mermoud A, Underdahl, Schnyder CC. An ultrasound biomicroscopic study of eyes after deep sclerectomy with collagen implant. Ophthalmology 1998; 105(4): 746-750.

17 Fiscella RG, Geller GL, Gryz LL, Wilensky J, Viana M. Cost considerations of medical therapy for glaucoma. Am J Ophthalmol. 1999; 128(4): 426-433.

18 Ashburn Jr FS, Goldberg I, Kass MA. Compliance with ocular therapy. Surv Ophthalmol 1980; 24(4): 237-248.

19 Migdal C, Gregory W, Hitchings R. Long term functional outcome after early surgery compared with laser and medicine in open-angle glaucoma. Ophthalomology 1994; 101: 1651-1656.

20 Mermoud A, Schnyder CC, Sickenberg M, Chiou AG, Hediguer SE, Faggioni R. Comparison of deep sclerectomy with collagen implant and trabeculectomy in open-angle glaucoma. J Cataract Refract Surg 1999; 25(3): 323-331.

21 El Sayyad F, Helal M, Khalil M, Schnyder CC, Faggioni R, Mermoud A et al. Nonpenetrating deep sclerectomy versus trabeculectomy in bilateral open-angle glaucoma. Ophthalmology 2001; 108(10): 1718

22 Sanchez E, Schnyder CC, Mermoud A. Comparative results of deep sclerectomy transformed to trabeculectomy and classical trabeculectomy. Klin Monatsbl Augenheilkd 1997; 210(5): 261-264.

23 Jenkins HP, Hrdina LS, Owens FM, Swisher FM. Absorbtion rates of different brands of chromic catgut. Arch Surg Chicago 1942; 45: 74.

24 Lawrie P. Studies in the absorption of surgical catgut. Ph.D Thesis, Edinburgh University Edinburgh: W Blackwood \& Sons Ltd, 1955. 